\title{
Combined Lesions of Hippocampus and Subiculum Do Not Produce Deficits in a Nonspatial Social Olfactory Memory Task
}

\author{
Stephen Burton, David Murphy, Uzma Qureshi, Patrick Sutton, and John O'Keefe \\ Department of Anatomy and Developmental Biology, University College London, London WC1E 6BT, United Kingdom
}

\begin{abstract}
Rats transmit information to each other about which foods are safe to eat. If a rat smells a food odor on the breath of another rat, it is subsequently more likely to eat that food than an alternative. Work by Galef et al. (1988) has shown that the observer rat forms an association between two olfactory stimuli on the breath of the demonstrator rat that has eaten the food, the food odor and carbon disulphide, which is normally present in the rat breath. Bunsey and Eichenbaum (1995) claimed that the hippocampus/ subicular region is required for the long-term retention of this nonspatial form of associative memory on the basis that combined lesions of the hippocampus and subiculum produced a deficit, but lesions of either structure alone did not. We report here a failure to repeat this finding. Rats with either combined
\end{abstract}

lesions of the hippocampus and subiculum or with amygdala lesions were tested on their ability to remember this association either immediately (testing short-term memory) or after a $24 \mathrm{hr}$ delay (testing long-term memory). Neither lesion group exhibited significant memory deficits on this nonspatial associative task at either test interval. In contrast, a deficit was observed on a spatial memory task (forced-choice alternation t-maze) for animals with combined lesions of the hippocampus and subiculum. These results contradict the findings of Bunsey and Eichenbaum (1995) and support the idea that the hippocampus/subicular region is not required for this nonspatial associative memory.

Key words: memory; social transmission; food preference; hippocampus; subiculum; amygdala; nonspatial
O'Keefe and Nadel (1978) have proposed a spatial mapping function (the "cognitive map theory") for the hippocampal formation. This theory predicts that lesions of the hippocampus will selectively disrupt spatial tasks but will leave nonspatial tasks unaffected. In general, results are broadly in support of this theory (Barnes, 1988). Jarrard (1993) reported that rats with selective neurotoxic lesions of the hippocampus exhibited only spatial memory deficits on the Olton radial arm maze, performing normally on nonspatial memory tasks. There are, however, a few reports of nonspatial deficits after hippocampal damage that are more consonant with a more generalized hippocampal function in the rat (Cohen and Eichenbaum, 1991, 1993). A good example is the finding by Bunsey and Eichenbaum (1995) that rats with combined hippocampus and subiculum neurotoxic lesions exhibited a deficit in a nonspatial associative memory task. This latter study therefore presents a strong challenge to the claim of cognitive mapping theory.

Bunsey and Eichenbaum (1995) used an adaptation of the social transmission of food preference model (Galef and Wigmore, 1983). In this three-stage experiment, a demonstrator rat first eats a scented food. Next, an observer rat interacts with the demonstrator during which it forms an association between two nonspatial olfactory stimuli on the demonstrator's breath (the food odor previously eaten and carbon disulphide) (Galef et al., 1988). Finally, when given a choice between that food and another not recently eaten by the demonstrator, the observer will preferentially eat the demonstrator's food, even after a delay of $24 \mathrm{hr}$ or more.

Winocur (1990) reported that rats with electrolytic lesions of the hippocampus exhibited no memory deficit on this task after a $24 \mathrm{hr}$ delay. Bunsey and Eichenbaum (1995) tested three lesion groups of rats with neurotoxic lesions restricted to the hippocampus, subiculum, and the hippocampus and subiculum combined. Like Winocur (1990), they found no memory deficit in animals with purely hip-

\footnotetext{
Received Feb. 22, 2000; revised April 19, 2000; accepted April 20, 2000.

The work was supported by a program grant from the United Kingdom Medical Research Council to J.O'K. We thank Dr. Kate Jeffrey and Colin Lever for commenting on an earlier draft of this paper. We also thank Drs. Fitzgerald and C. Yeo for help with histology.

Correspondence should be addressed to Prof. John O'Keefe, Department of Anatomy and Developmental Biology, University College London, Gower Street, London WC1E 6BT, UK. E-mail: j.okeefe@ucl.ac.uk.

Copyright (C) 2000 Society for Neuroscience $0270-6474 / 00 / 205468-08 \$ 15.00 / 0$
}

pocampal lesions, nor in the subiculum lesion group after a $24 \mathrm{hr}$ delay. However, they did report that the combined lesion group exhibited a significant memory deficit after the $24 \mathrm{hr}$ delay.

Here we report our attempts to reproduce the observations of Bunsey and Eichenbaum (1995). A total of five experiments were performed. In experiment 1, we evaluated rats' preference for the food flavors (cinnamon, clove, garlic, thyme, cocoa, marjoram, turmeric, and cumin) used. Experiment 2 looked at combined lesions of the hippocampus/subiculum in this nonspatial associative memory task. Failing to find an effect, we repeated the experiment with procedural modifications on new groups of rats in experiments 3 and 4 and investigated the possible role of the amygdala as well. Again, lesion groups showed no deficits. Experiment 5 was a test of spatial memory using the same animals from experiments 3 and 4 . We used the forced-choice alternation t-maze and found, as expected, a selective deficit in the animals with hippocampal formation damage, confirming the adequacy of the lesions. Histology verified the adequacy of the lesions. These results show that animals can successfully perform this nonspatial memory task, even without the hippocampus and subiculum.

\section{MATERIALS AND METHODS}

Subjects. One hundred twenty male Long-Evans rats (Harlan, Bicester, UK) weighing between 275 and $325 \mathrm{gm}$ at the start of testing were used in the five experiments. In each experiment, animals were divided into lesion groups receiving neurotoxic lesions and two control groups, an operated control (OC) group in which the operation was similar to that in the lesioned animals except the pipette was only lowered into the overlying cortex and no toxin was injected, and a suture control (SC) group in which the skin was incised and then resutured without any drilling of the skull. The external appearance of this latter group of animals was thus similar to that of the other operated groups. All animals were housed separately in $51 \times 31 \times 20 \mathrm{~cm}$ plastic bottom cages with sawdust bedding and kept on a 14/10 hr light/dark cycle. Lights came on at 7:00 A.M., and testing was done between 11:00 A.M. and 3:00 P.M.

Surgery. All surgical operations were performed before behavioral testing. Subjects were anesthetized with a mixture of isoflurane (1-chloro2,2,2-trifluoromethyl ether; Rhodia, Bristol, $\mathrm{UK}), \mathrm{N}_{2} \mathrm{O}$, and $\mathrm{O}_{2}$ and given a $0.1 \mathrm{ml}$ injection of the analgesic buprenorphine $(0.3 \mathrm{mg} / \mathrm{ml}$; Vetergesic Animal Care, York, UK) and then mounted in a Baltimore stereotaxic frame. The scalp was shaved and surgically cleaned. A midline incision exposed the skull. For the lesion and OC groups, the skull overlying the target area was removed with a trephine drill (Hager \& Meisinger, Dusseldorf, Germany). Bilateral injections of ibotenic acid $[10 \mu \mathrm{g} / \mu \mathrm{l}, \mathrm{pH} 7.4$ (Sigma, Poole, UK)] were made by pressure injection using coordinates 
given by Jarrard (1989) for hippocampus and subiculum lesions. Coordinates for the amygdala lesions were derived on the basis of pilot lesions [anteroposterior (AP), $-2.10 \mathrm{~mm}$; mediolateral (ML), -4.20 and -4.80 $\mathrm{mm}$; dorsoventral (DV), $-8.20 \mathrm{~mm}$; volumes, 0.15 and $0.05 \mu \mathrm{l}$; AP, -2.40 $\mathrm{mm}$; ML, $-4.20 \mathrm{~mm}$; DV, $-7.0 \mathrm{~mm}$; volume, $0.15 \mu \mathrm{l}$; AP, $-2.80 \mathrm{~mm}$; ML -4.40 and $-5.0 \mathrm{~mm}$; DV, $-6.90 \mathrm{~mm}$; volume, $0.15 \mu \mathrm{l}$; AP, $-3.30 \mathrm{~mm}$; ML, $-4.90 \mathrm{~mm}$; DV,$-7.20 \mathrm{~mm}$; volume, $0.15 \mu \mathrm{l}$. For the OCs, the pipette was lowered $0.50 \mathrm{~mm}$ into the neocortex at the same AP and ML coordinates, but no injection was performed. The SC group received a midline incision followed by suturing. All animals received a $0.1 \mathrm{ml}$ subcutaneous injection of enrofloxacin $(25 \mathrm{mg} / \mathrm{ml}$; Baytril Bayer, Suffolk, UK) to protect against post-operative infection, and all except the SC group received a 3-10 m injection of physiological saline to replace fluid lost during the operation. After recovery from surgery, amygdala-lesioned animals (A) received a $0.25 \mathrm{ml}$ intraperitoneal injection of diazepam $(5 \mathrm{mg} / \mathrm{ml}$; Phoenix Pharmaceuticals, Gloucester, UK) to prevent seizures; no seizures were observed with other surgical groups. Behavioral testing began 2 weeks after surgery. Histological analysis. At the end of behavioral testing, subjects received lethal injections of $0.5 \mathrm{ml}$ of sodium pentobarbitone $(200 \mathrm{mg} / \mathrm{ml}$, Euthatal; Rhône Mérieux, Essex, UK) and were perfused with physiological saline and $4 \%$ paraformaldehyde (Merck, Dorset, UK). The brains were embedded in gelatin before $40 \mu \mathrm{m}$ sections were cut on a cryostat (Bright, Huntingdon, UK). The brains of the amygdala-lesioned and OC-A groups were sectioned coronally, and those of the hippocampal/subiculum $(\mathrm{H} / \mathrm{S})$ and $\mathrm{OC}-\mathrm{H} / \mathrm{S}$ groups were sectioned horizontally. All sections were stained with cresyl violet. The extent of the damage was assessed using image analysis software (Leica Quantitative Interactive Programming System; Leica, Nussloch, Germany). The area of the amygdala and hippocampus subiculum was calculated by measuring every 10 th section of a normal rat brain. Structural volume was calculated by multiplying the sum of the areas by $0.40 \mathrm{~mm}$ (the distance between the sections). The size of the lesions were calculated by measuring the volume of remaining tissue (tissue that may not have been functional was included, thus preventing overestimation of lesion size). The percentage of brain tissue removed was calculated by subtracting this value from the average volume obtained by measuring five control brains.

Behavioral data analysis. The results were analyzed using repeated measures ANOVA for the effects of experimental group, delay interval, and the interaction between these two variables. After any indication of significant differences, planned $t$ tests were performed to evaluate specific effects within or across groups. Error bars in the graphs represent SEM.

Behavioral testing: shaping. One week before testing, all subjects were food deprived to $85 \%$ of free-feeding body weight and shaped to eat powdered rat diet (Harlan Teklad TRM 9607) from a feeding platform placed within their cage. The feeding platform consisted of a Plexiglas base $(25 \times 10 \times 0.3 \mathrm{~cm})$ with two clay pots (top diameter of $10 \mathrm{~cm}$, bottom diameter of $5 \mathrm{~cm}$ ) permanently fixed to the base $1 \mathrm{~cm}$ apart with a plastic (50 gm food capacity) cup secured within each clay pot. Food that was spilled but not eaten remained in the larger pot. Shaping was considered complete if an animal ate at least $1 \mathrm{gm}$ of powdered diet on 2 consecutive days (following the procedure of Bunsey and Eichenbaum, 1995).

\section{EXPERIMENT 1}

In the first experiment, we examined whether rats had systematic preferences for one of the two odors in each pair. A group of 12 male Long-Evans rats (Harlan) were food deprived to $85 \%$ of free-feeding body weight. Each animal was given four $2 \mathrm{hr}$ preference sessions in each of which they were allowed to choose between two flavored foods [cocoa $(8 \% \mathrm{w} / \mathrm{w}$ in powdered diet) versus cinnamon $(4 \% \mathrm{w} / \mathrm{w})$; clove $(1 \% \mathrm{w} / \mathrm{w})$ versus garlic $(0.8 \% \mathrm{w} / \mathrm{w})$; thyme $(4 \% \mathrm{w} / \mathrm{w})$ versus marjoram $(8 \% \mathrm{w} / \mathrm{w})$; or turmeric $(3 \% \mathrm{w} / \mathrm{w})$ versus cumin $(1.6 \% \mathrm{w} / \mathrm{w})]$. The pairings are those used in the original Bunsey and Eichenbaum (1995) study but the ratio of flavoring/diet constitutes a fourfold increase.

\section{Results}

Eleven of 12 rats preferred cinnamon over cocoa and clove over garlic, (a different animal liked cocoa from the one that liked garlic), but only 6 of 12 had a preference for thyme and turmeric. Figure 1 shows these preferences in terms of the average percentage of each food eaten.

Despite these imbalances, we decided to use the same pairings in our experiments as those used by Bunsey and Eichenbaum (1995) to maintain comparability. Cocoa was paired with cinnamon, cumin with marjoram, turmeric with thyme, and clove with garlic. However, to minimize adventitious results caused by the natural preferences of the rats, in experiment 2 , the least preferred food of each pair was designated as the food to be fed to the demonstrator (hereafter called the target food).

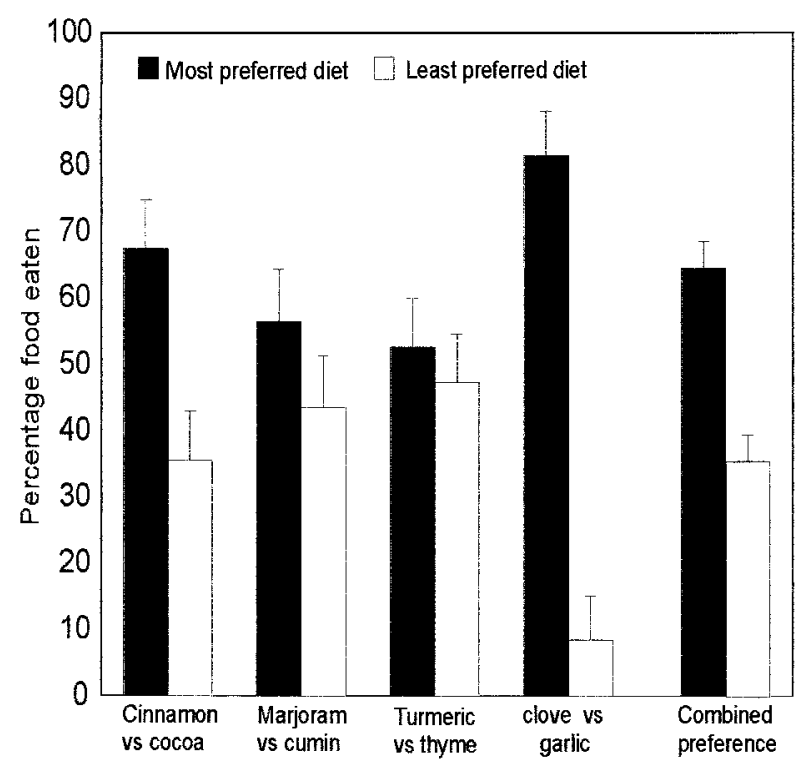

Figure 1. Average preference of 12 normal control animals for each flavor within a pair.

\section{EXPERIMENT 2}

Thirty-six naïve Long-Evans rats forming 18 observer-demonstrator pairs were used in experiment 2. Nine observer rats received combined bilateral lesions of the hippocampus and subiculum (group $\mathrm{H} / \mathrm{S}$ ), and nine animals formed the control group, six SCs and three OC-H/Ss.

After surgery, the experiment was run blind. Each animal was coded and remarked, and the experimenters who tested the animals were unaware of the group to which each animal belonged. The demonstrator and observer rats were kept at $85 \%$ of initial body weight throughout the experiment. Before training, the observer and demonstrator from each pair were allowed to interact while separated by a wire mesh screen for $4 \mathrm{hr}$ on 4 consecutive days to develop familiarity with each other. They were then shaped to eat from the food bowls, followed by experimental testing. Each observer received four different testing sessions. During each session, a different flavored food pairing was tested. In each session, two choice bowls were placed in different locations within the observer's cage. On two of these sessions, the observer was allowed an immediate choice after interacting with the demonstrator, whereas on the remaining two sessions, there was a $24 \mathrm{hr}$ delay before the choice. Each testing interval was randomized for all subjects, and there was a delay of $1 \mathrm{~d}$ between each testing session. The demonstrator rats were always fed the least preferred food (as determined from experiment 1) of the food pairs. The demonstrator was allowed to eat the target food for $30 \mathrm{~min}$. If the demonstrator did not eat at least $1 \mathrm{gm}$ of the target food at this stage, the data from that trial were discounted. Immediately after this stage, each demonstrator rat was allowed to interact with its paired observer for 20 min and then returned to its own home cage. Either immediately (0 delay) or after a $24 \mathrm{hr}$ delay, the observer rat was presented with the target food together with the alternative and given $2 \mathrm{hr}$ to eat either of the two foods. Both food bowls were weighed before and after feeding, and the percentage of the target food eaten was calculated as (target food eaten/total of both foods eaten) $\times 100$.

\section{Results}

Both controls and lesion animals chose the target food significantly more often than would be expected by chance at both intervals. The target food was preferred on 13 of 16 occasions (four pairs $\times$ 2 delays $\times 2$ groups). The lesion group ate a greater proportion of the target food than did the controls on 7 of 8 occasions (Fig. 2). ANOVA indicated a significant difference in performance between groups $\left(F_{(1,32)}=8.71, p<0.01\right)$ because of the $\mathrm{H} / \mathrm{S}$ lesion group 


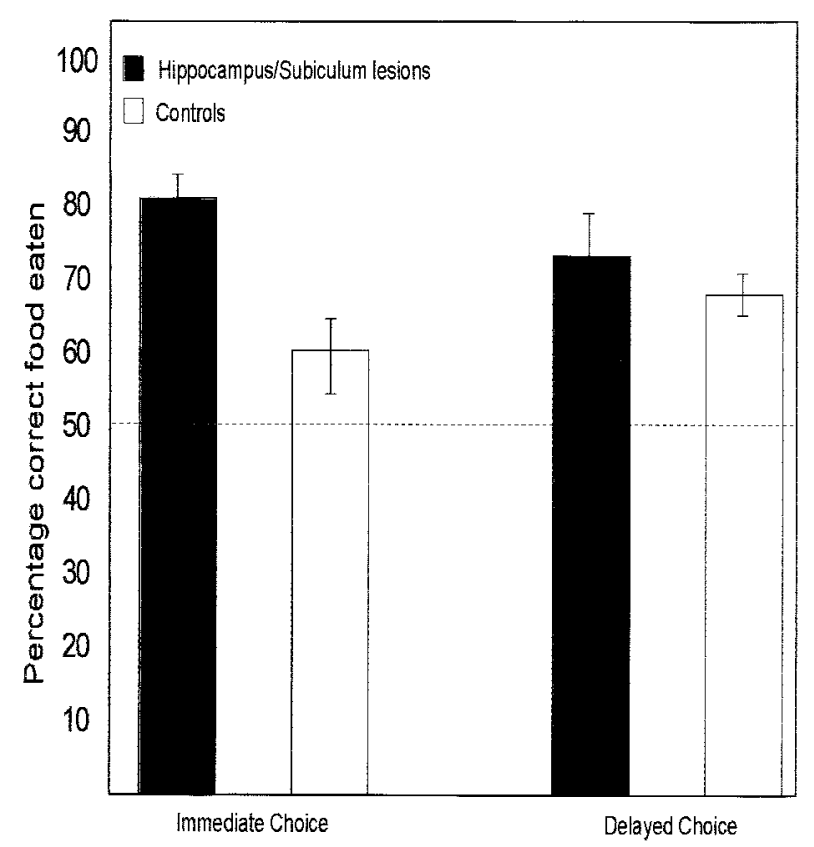

Figure 2. Average percentage of the target food eaten by the lesion group and the control animals either immediately (left) or after a $24 \mathrm{hr}$ delay period (right) in experiment 2.

performing better overall than the controls. No significant differences were observed when comparing delay periods $\left(F_{(1,32)}=0.01\right.$, $p>0.05$, NS) or delay period by group interaction $\left(F_{(1,32)}=3.27\right.$, $p>0.05, \mathrm{NS})$. A subsequent $t$ test indicated a significantly better performance of group $\mathrm{H} / \mathrm{S}$ over the controls $(t=3.08, p<0.01)$.

\section{Comments}

All observer groups showed a preference for the flavor of the target food fed to the demonstrator at both zero and $24 \mathrm{hr}$ delay, reversing the natural tendency seen in experiment 1 . Furthermore, there was no hint of a memory deficit in the lesion group at either delay. If anything, the $\mathrm{H} / \mathrm{S}$ lesion group demonstrated superior memory. This finding contrasts with that of Bunsey and Eichenbaum (1995), who found a deficit in their H/S group after a $24 \mathrm{hr}$ delay.

\section{EXPERIMENT 3}

Given our failure to replicate the original Bunsey and Eichenbaum (1995) results, we compared the details of our protocol with theirs and examined possible explanations for the discrepancy. We noted that they had not assessed the natural preference of rats for the odor pairs used but had controlled for any bias by counterbalancing the target odor across animals. Second, we used a wire screen to separate the demonstrator and observer animals as had Winocur (1990) to reduce aggressive interactions between them, whereas Bunsey and Eichenbaum had not. Finally, we wondered whether their lesions had inadvertently damaged the nearby amygdala or fibers arising from that structure. In the two next replication experiments, we altered the protocol to bring it closer to that of Bunsey and Eichenbaum, and we also included an amygdala lesion group.

Experiment 3 was a replication of experiment 2 with a new group of naïve animals except for two differences. First, we used the same four flavored diet pairings that were used in experiment 2 , but now the target food within each pair was not the least preferred one but was counterbalanced within each group. Second, we added a group with bilateral amygdala lesions to test the involvement of this structure. As in experiment 2, the experimenters doing the behavioral testing were blind to the animals' group.

\section{Subjects}

The subjects of experiment 3 (and 4, see below) consisted of 72 male Long-Evans rats separated into 36 demonstrator-observer

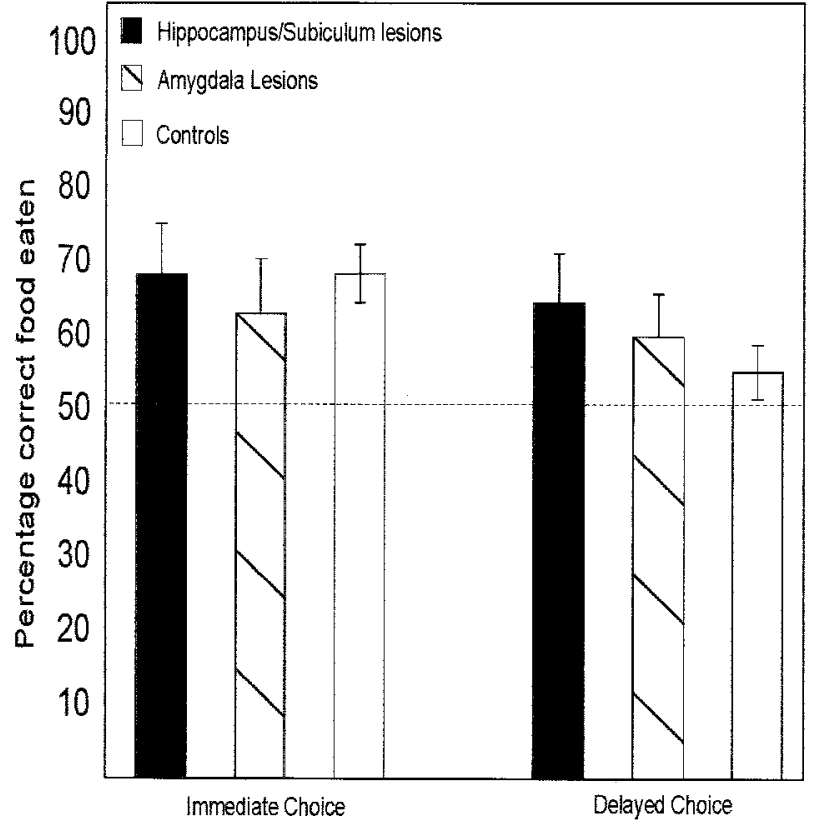

Figure 3. Average percentage of the target food eaten by the three groups either immediately (left) or after a $24 \mathrm{hr}$ delay period (right) in experiment 3 .

pairs. The H/S group was composed of twelve observer animals. Another twelve observers received bilateral amygdala lesions (group A). Twelve animals formed the control group, eight receiving operated control lesions (groups OC-A, $n=4$; OC-H $/ \mathrm{S}, n=4$ ) and four serving as SCs. All were food deprived to $85 \%$ of free-feeding body weight.

\section{Results}

The performance of the animals in the suture control group was slightly, but not significantly, inferior to the operated controls and, for the purpose of the ANOVA, the two control groups were combined. All groups chose the target food significantly more often than expected by chance (all $t$ values $>1.94, p<0.05$ ) with the exception of the suture controls after the $24 \mathrm{hr}$ delay $(t=0.39$, NS). There were no significant differences between any of the groups at either delay period (Fig. 3) because all groups performed at an equivalent level. A two-factor ANOVA (group $\times$ delay period) with replication showed no significant differences between groups $\left(F_{(2,66)}=0.444, p>0.05, \mathrm{NS}\right)$ or delay period $\left(F_{(1,66)}=1.99, p>\right.$ $0.05, \mathrm{NS})$ or delay period $\times$ group interaction $\left(F_{(2,66)}=0.475, p>\right.$ $0.05, \mathrm{NS})$.

\section{Comments}

The results of experiment 3 again showed no significant evidence of a memory deficit for the $\mathrm{H} / \mathrm{S}$ group, again failing to replicate the observations of Bunsey and Eichenbaum (1995). Furthermore, there was no evidence of a deficit in the amygdala lesion group.

Two additional minor differences in our protocol from that used by Bunsey and Eichenbaum (1995) were our use of a wire screen to separate the animals during their social interaction and the fact that our animals were hungrier than theirs because they were on food deprivation. It is possible that increased hunger reduces the animals' preference for the target food. To eliminate these discrepancies, experiment 4 followed the protocol of Bunsey and Eichenbaum in not using a screen and in not depriving the observer rats of food before testing.

\section{EXPERIMENT 4}

\section{Subjects}

The subjects were the same as those used in experiment 3 , but the demonstrator-observer pairings were changed. The experimenters 


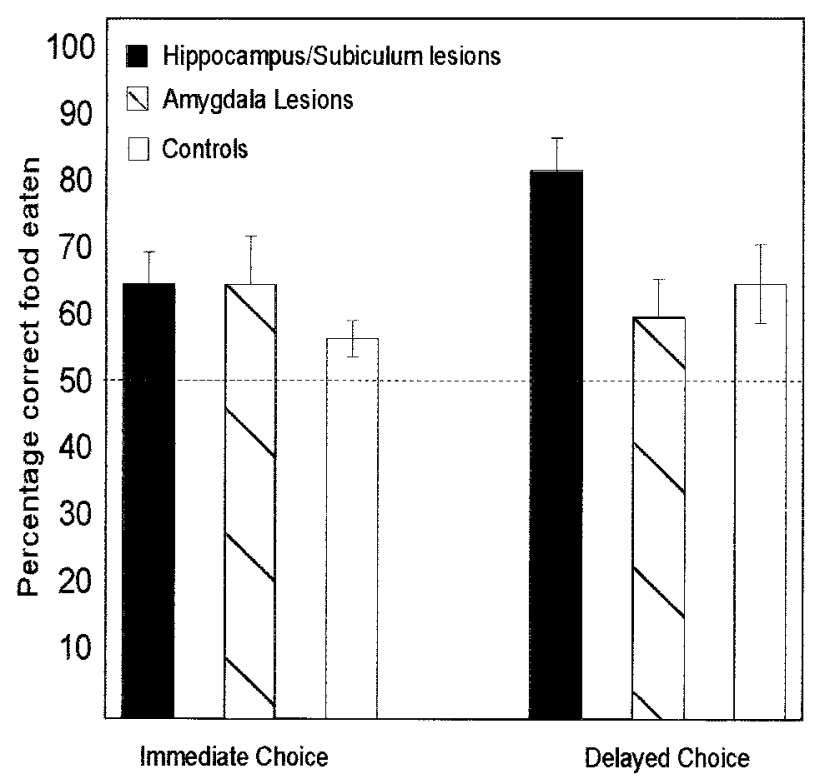

Figure 4. Average percentage of the target food eaten either immediately (left) or after a $24 \mathrm{hr}$ delay period (right) in experiment 4.

were once again ignorant of the group affiliation of the subjects. There were two differences in procedure between experiments 3 and 4. In experiment 4, the demonstrators were still food deprived, but the observers had ad libitum access to food and water. To allow more open interaction between the pairs, the wire mesh screen was removed. To allow for familiarization and to prevent or reduce fighting, the new observer-demonstrator pairs were housed together for 1 week before testing.

\section{Results}

All groups performed significantly better than chance when tested immediately $(\mathrm{t}>1.98, p<0.05)$. When tested after a $24 \mathrm{hr}$ delay, all groups performed significantly better than chance $(\mathrm{t}>2.22, p<$ $0.025)$ with the exception of the amygdala group $(t=1.669, p>$ $0.05)$. As seen in Figure 5, the change in the food deprivation status of the observer rats resulted in a marked decrease in the total amount of food eaten compared with experiment 3. Despite this, there was no evidence of a deficit in the H/S animals (Fig. 4). If anything, these animals showed a greater preference for the target food then did the controls (Fig. 4). A two-factor ANOVA (group $\times$ delay period) with replication indicated a significant difference between groups $\left(F_{(2,66)}=3.32, p<0.05\right)$. There were no significant differences for the delay period $\left(F_{(1,66)}=3.09, p>0.05, \mathrm{NS}\right)$ or delay period $\times$ group interaction $\left(F_{(2,66)}=1.85, p>0.05\right.$, NS $)$. Subsequent $t$ tests confirmed that the $\mathrm{H} / \mathrm{S}$ animals performed significantly better than the control group $(t=2.5, p<0.02)$ and the amygdala group $(t=2.6, p<0.02)$ but that the control and amygdala groups did not differ $(t=0.578, p=0.57$, NS).

\section{Comments}

As in experiments 2 and 3, group $\mathrm{H} / \mathrm{S}$ failed to show a memory impairment. Moreover, in experiment 4, the $\mathrm{H} / \mathrm{S}$ group performed significantly better than the controls. Groups on average overall ate less in experiment 4 then they had in experiment 3, ruling out level of motivation as an important variable (Fig. 5). The continued absence of a deficit on this nonspatial memory task prompted us to ask whether our lesions were of a sufficient quality to produce memory impairment. To test this question, we tested the performance of the animals from experiments 3 and 4 on a spatial working memory task.

\section{EXPERIMENT 5}

\section{Subjects}

The 36 observer animals from experiments 3 and 4 were used. Two animals failed to pass the pretraining stage, and they were elimi-

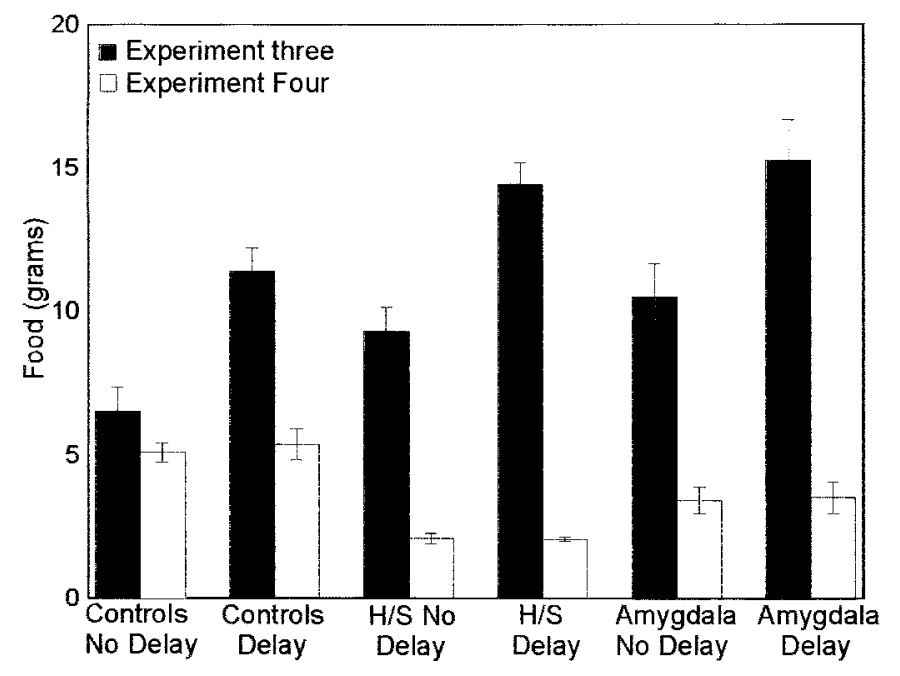

Figure 5. Average total amount of food eaten in experiments 3 and 4.

nated from the study. One of these was from the H/S group and the other from the control group. An additional animal from the control group continually jumped off the t-maze and was therefore removed from the study. The final numbers in each group were 11 $\mathrm{H} / \mathrm{S}, 12 \mathrm{~A}$, and 10 control animals.

\section{Apparatus: forced-choice alternation t-maze}

The elevated maze was made of Perspex and wood painted black and located in a central position in a room $(3.5 \times 2.9 \mathrm{~m})$, which also contained the animals' home cages against the west wall and a table against the north wall. The start arm and the reward arms of the t-maze were $14 \mathrm{~cm}$ wide and $38 \mathrm{~cm}$ long, with a $34 \mathrm{~cm}$ diameter central section connecting the reward arms. The whole maze was raised $32 \mathrm{~cm}$ off the ground. Food wells $4 \mathrm{~cm}$ in diameter and $0.7 \mathrm{~cm}$ deep were situated at the end of reward arms.

\section{Procedure}

Testing commenced 2 weeks after completion of experiment 4 . Animals received $5 \mathrm{~d}$ of pretraining during which they learned to run down the start arm and eat food pellets from the food wells at the end of the reward arms. The testing phase of the experiment consisted of a series of trials, each consisting of two runs in the maze: an information run and a test run. On the information run, a wooden block $(30 \times 18 \times 9 \mathrm{~cm})$ was placed at the start of a reward arm. This obstruction forced the rat to enter the other reward arm where it found three $45 \mathrm{mg}$ food pellets (Camden Instruments, London, UK) in the food well. The subject was given time to eat the reward and then placed in a holding box for 1 min before starting the second run. On the test run, the block was removed, and the animal had to enter the arm previously unvisited to receive a food reward. A choice was considered to have been made when the rat placed either of its hind feet into an arm. If after $60 \mathrm{sec}$ the rat failed to enter an arm on either run, then that trial was not included in the data. After the animal made its choice, the entrance to the chosen arm was blocked off to prevent backtracking. If the rat entered the wrong arm, it was allowed to go to the end of the arm (where it did not receive a food reward). The rats were tested in groups of four, each rat having one trial in turn. This resulted in an intertrial interval ranging from 12 to $15 \mathrm{~min}$. Each rat received six trials per day (three forced left and three forced right) for 12 consecutive days. The order of trials for each day was determined by a pseudorandom schedule with no more than three consecutive left or right trials (Fellows, 1967).

\section{Results}

Across the entire 72 trials, the controls averaged $73 \%$ correct, the amygdala lesioned animals averaged $70 \%$ correct, and the $\mathrm{H} / \mathrm{S}$ group averaged $49 \%$ correct (Fig. 6). A single-factor ANOVA 


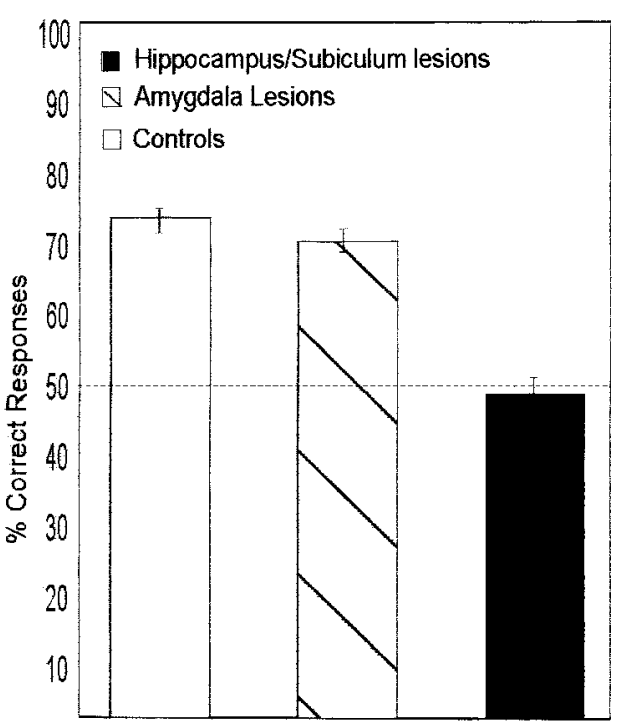

Figure 6. Mean correct scores of animals with hippocampus/subiculum lesions, amygdala lesions, and controls on the forced-choice spatial alternation in experiment 5 .

indicated a significant difference between groups $\left(F_{(2,30)}=19.26\right.$ $p<0.0001)$. Subsequent post hoc $t$ tests indicated a significant deficit in the performance of $\mathrm{H} / \mathrm{S}$ animals when compared with the performance of control animals $(t=7.79, p<0.0001)$ and group A $(t=4.56, p<0.001)$. The performance of group $\mathrm{A}$ is below that of the control group, although not significantly so $(t=$ $0.78, p>0.05$, NS.).

\section{Comments}

The same animals with hippocampal/subiculum damage, which had normal or superior performance on the social olfactory association task, were profoundly deficient on a one-trial spatial memory task. The results from experiment 5 illustrate that the lesions of the $\mathrm{H} / \mathrm{S}$ group were of a sufficient quality to produce spatial memory deficits. Therefore the absence of any deficits in the two previous nonspatial associative memory experiments is unlikely to have been attributable to ineffective lesions. This was subsequently confirmed histologically.

\section{Histological analysis}

In all subjects of the $\mathrm{H} / \mathrm{S}$ groups, ibotenic acid lesions produced extensive damage of the hippocampus and subiculum throughout their dorsoventral and anteroposterior fields. From the measurement of five normal brains, a volume of $112 \mathrm{~mm}^{3}$ (range of 106-118 $\mathrm{mm}^{3}$, bilateral total) was calculated for the hippocampus and subiculum, and a volume of $22 \mathrm{~mm}^{3}$ (range of $19-24.5 \mathrm{~mm}^{3}$, bilateral total) was calculated for the amygdala. From these, the percentage tissue loss in the lesioned animals was calculated. The animals of experiment 2 sustained an average of $85 \%$ (range of 55-99\%) tissue loss to the hippocampus and subiculum. The 12 $\mathrm{H} / \mathrm{S}$ animals of experiments 3-5 sustained a similar average $85 \%$ volume loss (range of 42-98\%) (Fig. 7) in the hippocampus/subiculum. In both groups, the remaining hippocampal tissue occupied primarily the dentate gyrus subfields in the dorsal and ventral hippocampal formation. There was also extensive damage to the presubiculum, parasubiculum, and entorhinal cortex. Damage outside the hippocampal formation was similar in both groups and included neocortex, striatum, thalamus, and amygdala to varying extents. Moderate damage was sustained by several thalamic nuclei, primarily anterodorsal, anteroventral, and paraventricular nuclei. In addition, the centrolateral nuclei were damaged in some but not all animals, and minor damage was sustained by the laterodorsal, reticular, dorsolateral geniculate, medial geniculate, and posterior intralaminar thalamic nuclei. Moderate damage was found in the striatum and the lateral, medial, and central nuclei of the amygdala.

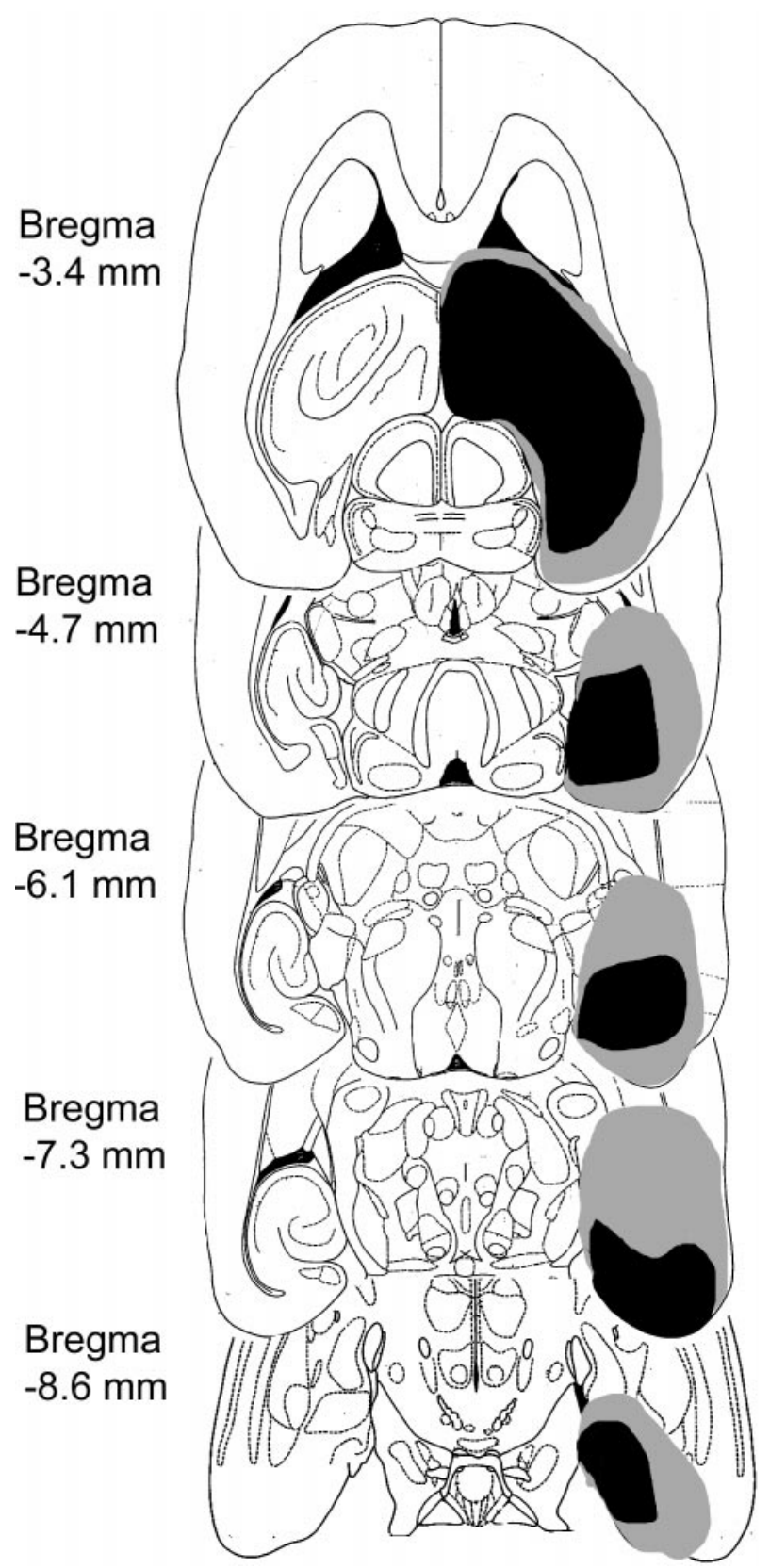

Figure 7. A reconstruction of the largest (grav) and smallest (black) hippocampus/subiculum lesions. Lesions of the left and right hemisphere are shown on the right. The drawings of horizontal sections are adapted from the atlas of Paxinos and Watson (1986).

In addition, light damage was seen in the following structures: piriform, temporal, perirhinal, retrosplenial, occipital, parietal and frontal cortices, dorsal and ventral endopiriform nuclei, anterior cortical and basal nuclei of the amygdala, and the habenula. A representative animal from experiments 3-5 with $90 \%$ of hippocampus/subiculum damage is shown in Figure $8, C$ and $D$. There were no significant correlations between the size of the lesion and the performance of the hippocampus/subiculum group in any experiment.

Ibotenic acid lesions in the amygdala produced extensive damage to all amygdala nuclei, resulting in an average volume loss of $79 \%$ (range of 54-98\%), which is shown in Figure 9. Cell and tissue loss was also extensive in the striatum and the piriform, parietal, perirhinal, and entorhinal cortices, with partial loss to the dorsal and ventral endopiriform nuclei, claustrum, and the dorsal and ventral hippocampus. An animal from experiments 3-5 with $54 \%$ of amygdala damage is shown in Figure 10. 

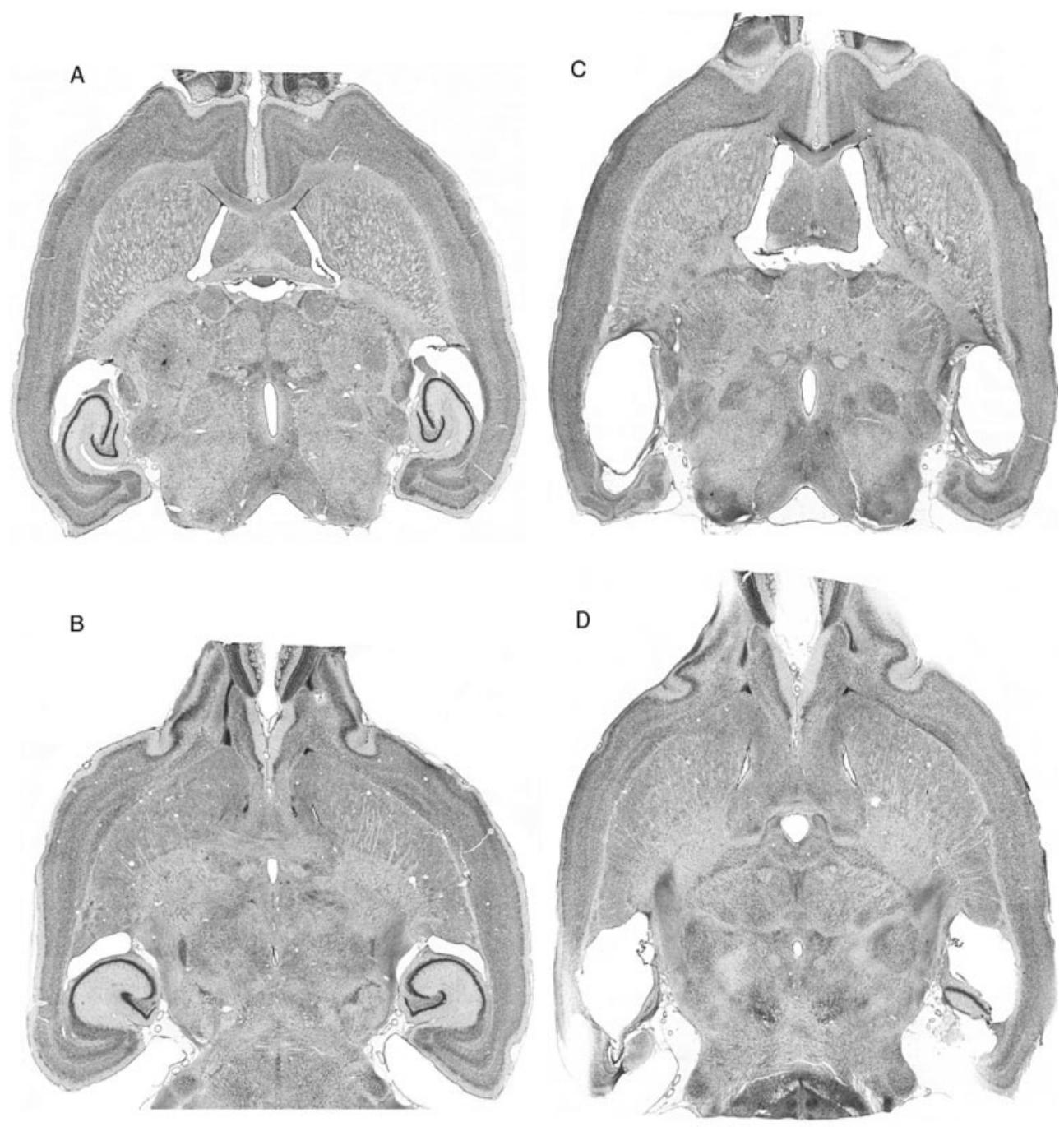

$2 \mathrm{~mm}$

Figure 8. Representative lesion from the hippocampus/subiculum group in experiments 3-5 $(C$, $D)$ with a control for comparison $(A, B)$.

\section{DISCUSSION}

In the present experiments, we have not found a deficit in the learning or retention of a nonspatial olfactory stimulus-stimulus association after combined damage to the hippocampus and subiculum. In contrast, the same animals failed to learn a one-trial spatial memory task. Lesions of the amygdala did not impair the learning or the retention of the olfactory association or of the spatial task. These results stand in contrast to those of Bunsey and Eichenbaum (1995) who found a deficit in their hippocampal formation animals after a $24 \mathrm{hr}$ delay. Bunsey and Eichenbaum presented their results as strong evidence for an important role for the hippocampal formation in nonspatial associative tasks and therefore as a challenge to the cognitive map theory of hippocampal function. They further noted that the olfactory social task was a good example of an ecologically valid task that tapped into natural learning abilities that an animal might be expected to use in its normal habitat. We agree with these points but conclude that our results support the cognitive map theory of hippocampal function.

We cannot account for the discrepancy between the findings of the present experiment and those of Bunsey and Eichenbaum (1995). In our pilot experiments (data not included), we found that the odor concentrations used by Bunsey and Eichenbaum were too low to provide consistent discriminations in our rats and increased them to obtain more consistent results. In our odor preference tests (experiment 1), we found that two of the pairs of odors used in the Bunsey and Eichenbaum study were relatively equally matched but that the other two pairs revealed strong and consistent preferences among our rats. We checked these results by giving the same group of rats all eight odors to choose among and obtained relative preferences which accorded with the results reported in experiment 1. Nevertheless, we decided to use the same pairings as Bunsey and Eichenbaum to maintain consistency with their experiment. It is possible, although unlikely, that our use of increased concentrations affected the relative odor preferences of our animals.

We do not think that the discrepancy in the two sets of results is attributable to procedural differences. In the three replications, we varied several of these and it made no difference. When two unfamiliar male rats are placed together, they sometimes fight. Bunsey and Eichenbaum (1995) did not mention observing this in their animals and did not make any provision for it. Our pilot experiments showed that it was a factor in our animals. To reduce this tendency, we followed Winocur's (1990) procedure of separating the demonstrator and observer rats by a wire screen during their interaction in experiments 2 and 3. In experiment 4, we dispensed with the screen but allowed the animals to interact with each other for 1 week before the experiment so that they would 


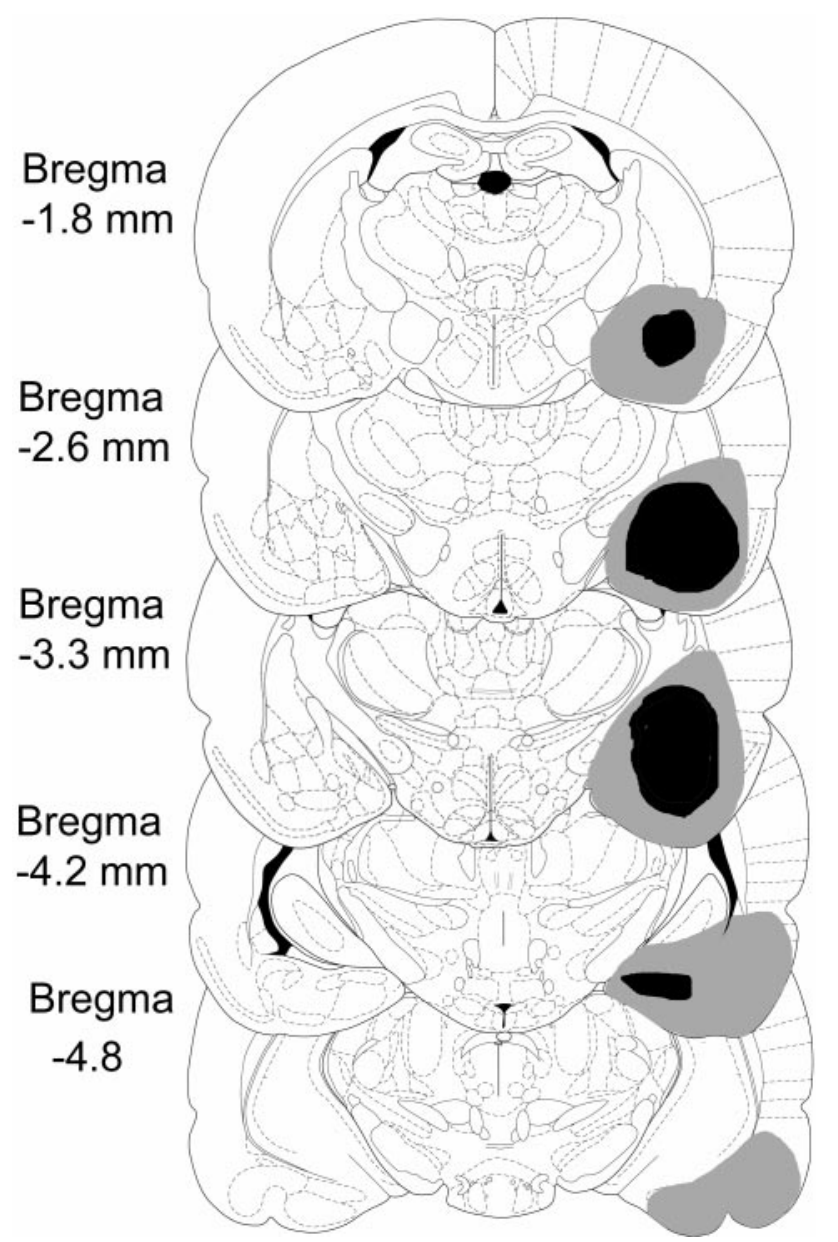

Figure 9. A reconstruction of the largest (gray) and smallest (black) amygdala lesions. Lesions of the left and right hemisphere are shown on the right. The drawings are adapted from the atlas of Paxinos and Watson (1997)

become familiar with each other. In neither case was there a deficit in the animals with hippocampal/subicular damage. We also considered the possibility that the level of food deprivation, and hence the animals' motivation, might be a factor. The observer rats in the Bunsey and Eichenbaum experiment were not food deprived, and we followed this procedure in experiment 4. In experiments 2 and 3 , however, our animals were food deprived and, as a result, ate considerably more food during the test phase. Comparison of the amount of food eaten by our animals and those of Bunsey and Eichenbaum shows that, on average, our hungry animals ate more than theirs and our sated animals less. Again, in neither of these conditions was there a deficit.

We went to considerable trouble to ensure that the experimenters who performed the behavioral testing were unaware of the animals' lesion status. In experiment 2, the allocation of animals to their respective groups and the operations were done by one of us (S.B.), with the behavioral testing performed by three different experimenters (U.Q., P.S., and D.M.) who were unaware of the group to which each animal belonged. In experiments 3 and 4, the animals were recoded and remarked after the operations by someone other than the experimenter. Furthermore, all control animals had scalp incisions so that they were indistinguishable in appearance from the operated groups. Only in experiment 5 was the lesion status of the animals known to the experimenter. This result is not contentious, however, because it has been found on many occasions previously either after lesions to the hippocampus itself or to its afferent and efferent targets (Aggleton et al., 1986, 1995; Neave et al., 1997; Bussey et al., 1998). The test was used here primarily to demonstrate the effectiveness of the lesions.
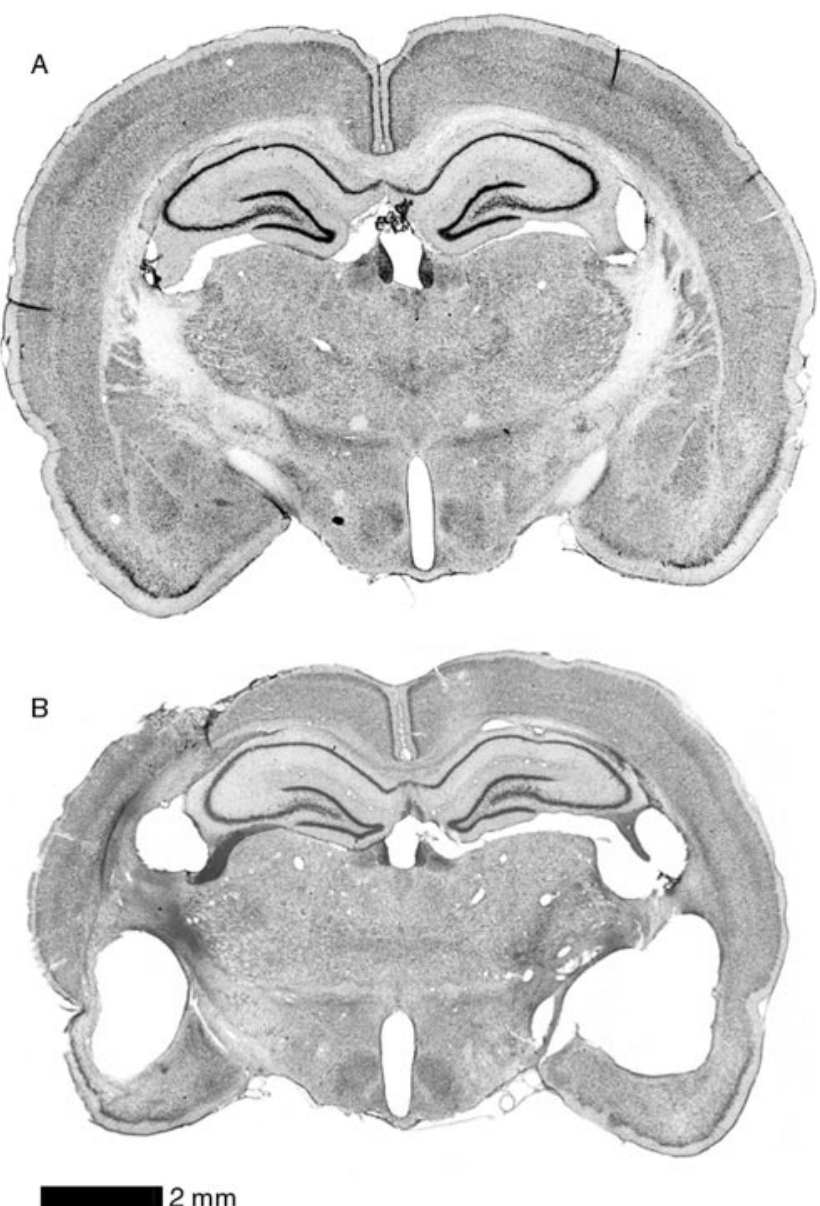

Figure 10. A lesion from amygdala group in experiments 3-5 $(B)$ with a control for comparison $(A)$.

Not only were animals with lesions of the hippocampus/subiculum not deficient relative to controls in the social transmission of olfactory information, but in two of the three tests (experiments 2 and 4), they ate significantly more of the target food than did the controls. The cognitive map theory predicts that, under circumstances in which a spatial hypothesis conflicts with successful performance in a task, animals with hippocampal lesions will perform better than normal controls. However, there is little evidence to suggest that rats normally entertain spatial hypotheses in the current task. Beck and Galef (1989) investigated whether the location where the demonstrator rat ate the food had any influence on the observer rats' choice and found none. In the present task, the two choice bowls were placed in different locations within the observer's cage on different trials, offering little opportunity for the development of place hypotheses. In light of these considerations, we can offer no cogent hypothesis as to why the hippocampal/ subicular animals might perform better on this task.

Finally, we consider the size and nature of the lesions as a possible difference between this experiment and previous experiments. Whereas Winocur (1990) used electrolytic lesions to damage the dorsal hippocampus, both Bunsey and Eichenbaum (1995) and ourselves used the neurotoxin ibotenic acid to make the lesions. It seems unlikely, therefore, that in either of these two latter experiments was there significant damage to fibers of passage from retrohippocampal structures as might have occurred in Winocur's experiment. Lesions to the combined hippocampal/subicular group in the Bunsey and Eichenbaum experiment damaged $84 \%$ of the hippocampus proper and dentate gyrus (range of 71-96\%) and $79 \%$ of the subiculum (range of 77-98\%). The combined damage to the hippocampus and subiculum of the rats in our experiment 2 was $85 \%$ (range of 55-99\%) and in experiment 3-5 was 85\% (range of 
42-98\%). Furthermore, the possibility that the Bunsey and Eichenbaum results were attributable to inadvertent damage to the amygdala complex can be ruled out by the absence of a deficit in our amygdala group. We conclude that the differences between the results of the present experiments and those of Bunsey and Eichenbaum are not attributable to differences in the lesions in the two experiments.

In conclusion, the results of the present study fail to replicate the Bunsey and Eichenbaum (1995) finding of a deficit in the social transmission of olfactory information after damage to the hippocampal/subicular region. Rather, the present results support the idea that the integrity of this part of the brain is not fundamental to the learning and retention of this nonspatial stimulus-stimulus association.

\section{REFERENCES}

Aggleton JP, Hunt PR, Rawlins JNP (1986) The effects of hippocampal lesions upon spatial and non-spatial tests of working memory. Behav Brain Res 19:133-146.

Aggleton JP, Neave N, Nagle S, Sahgal A (1995) A comparison of the effects of medial prefrontal, cingulate cortex, and cingulum bundle lesions on tests of spatial memory: evidence of a double dissociation between frontal and cingulum bundle contributions. J Neurosci 15:7270-7281.

Barnes CA (1988) Spatial learning and memory processes: the search for their neurobiological mechanisms in the rat. Trends Neurosci 11:163-169.

Beck M, Galef Jr BG (1989) Social influences on the selection of a protein-sufficient diet by Norway rats (Rattus norvegicus). J Comp Psychol 103:132-139.

Bunsey M, Eichenbaum H (1995) Selective damage to the hippocampal region blocks long-term retention of a natural and non-spatial stimulusstimulus association. Hippocampus 5:546-556.

Bussey TJ, Warburton C, Aggleton JP, Muir JL (1998) Fornix lesions can facilitate acquisition of the transverse patterning task: a challenge for "configural" theories of hippocampal function. J Neurosci 18:1622-1631.

Cohen NJ, Eichenbaum H (1991) The theory that wouldn't die: a critical look at the spatial mapping theory of hippocampal function. Hippocampus 1:265-268.

Cohen NJ, Eichenbaum H (1993) Memory, amnesia, and the hippocampal system. Cambridge, MA: MIT.

Fellows BJ (1967) Chance stimulus sequences for discrimination tasks. Psychol Bull 67:87-92.

Galef Jr BG, Wigmore SW (1983) Transfer of information concerning distant foods: a laboratory investigation of the "information-centre" hypothesis. Anim Behav 31:748-758.

Galef Jr BG, Mason RJ, Preti G, Bean JN (1988) Carbon disulfide: a semiochemical mediating socially-induced diet choice in rats. Physiol Behav 42:119-124.

Jarrard LE (1989) On the use of ibotenic acid to lesion selectively different components of the hippocampal formation. J Neurosci Methods 29:251-259.

Jarrard LE (1993) On the role of the hippocampus in learning and memory in the rat. Behav Neural Biol 60:9-26.

Neave N, Nagle S, Aggleton JP (1997) Evidence for the involvement of the mammillary bodies and cingulum bundle in allocentric spatial processing by rats. Eur J Neurosci 9:941-945.

O'Keefe J, Nadel L (1978) The hippocampus as a cognitive map. Oxford: Clarendon.

Paxinos G, Watson C (1986) The rat brain in stereotaxic co-ordinates. Toronto: Academic.

Paxinos G, Watson C (1997) The rat brain in stereotaxic co-ordinates. Toronto: Academic.

Winocur G (1990) Anterograde and retrograde amnesia in rats with dorsal hippocampal or dorsomedial thalamic lesions. Behav Brain Res 38: 145-154. 